\title{
Comparison of serum levels of asymmetric dimethylarginine between patients who take two types of atypical anti psychotics
}

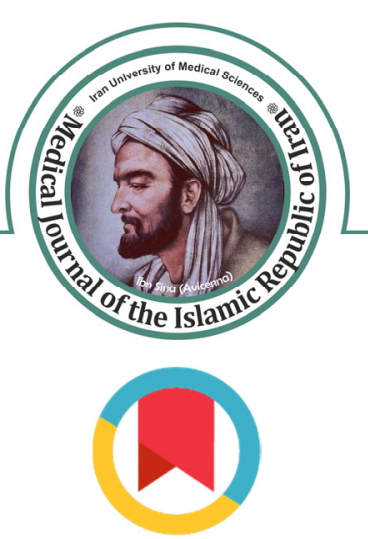

\section{Maryam Safaei ${ }^{1,2,3}$, Ali Akhondpoor Manteghi ${ }^{2}$, Najmeh Shahini ${ }^{4}$, Amir Hooshang Mohammadpour*5,6}

Received: 28 Apr 2017

Published: 23 Oct 2019

\section{Abstract}

Background: Schizophrenia is associated with increased cardiovascular morbidity. Asymmetric dimethylarginine (ADMA) has been suggested as a cardiovascular biomarker. Treatment with atypical antipsychotics can increase some traditional risk factors of coronary artery disease. In addition to traditional risk factors, this study is carried out as a comparison of serum levels of ADMA and non-traditional factors among patients who take two types of atypical antipsychotics.

Methods: In this clinical study, 57 schizophrenic patients with multiple episodes and 20 healthy voluntaries that fulfilled inclusion and exclusion criteria were entered into the study. The patients were divided into 3 groups (18 patients received risperidone alone, 20 patients received clozapine alone and 19 patients did not receive any drug). Plasma concentrations of ADMA, high-sensitivity Creactive protein (hs-CRP) and homocysteine were measured through enzyme-linked immunosorbent assay (ELISA), and traditional risk factors of metabolic syndrome were measured.

Results: Mean age of participants was $46.08 \pm 12.54$ years. Moreover, the traditional (High-density lipoprotein (HDL), total cholesterol, waistline, and Body Mass Index (BMI)) and non-traditional factors (Homocysteine, hs-CRP) and ADMA were higher in patients with schizophrenia compared to healthy group $(\mathrm{p} \leq 0.05)$. Also, in the clozapine group, all mentioned non-traditional factors and ADMA were significantly higher than other groups $(\mathrm{p} \leq 0.05)$.

Conclusion: In the clozapine group, levels of non-traditional factors and ADMA were significantly higher which indicates these patients are at risk of cardiovascular disease.

Keywords: Asymmetric dimethylarginine, Atypical antipsychotics, Metabolic syndrome, Schizophrenia

Conflicts of Interest: None declared

Funding: None

*This work has been published under CC BY-NC 4.0 license.

Copyright $($ Iran University of Medical Sciences

Cite this article as: Safaei M, Akhondpoor Manteghi A, Shahini N, Mohammadpour AM. Comparison of serum levels of asymmetric dimethylarginine between patients who take two types of atypical anti psychotics. Med J Islam Repub Iran. 2019 (23 Oct);33:114. https://doi.org/10.47176/mjiri.33.114

\section{Introduction}

Asymmetric dimethylarginine (ADMA) is a competitive inhibitor of nitric oxide synthase (NO) and inhibits the production of pathologic levels of vascular NO. Its intraarterial administration can lead to local vasoconstriction. ADMA has been suggested as a cardiovascular biomarker in previous studies, and its serum level was found to be increased in patients with coronary artery disease and

Corresponding author: Dr Amir Hooshang Mohammadpour, mohamadpoorah@mums.ac.ir

1. Bojnord University of Medical Sciences, Bojnord, Iran

2. Psychiatry and Behavioral Sciences Research Center, Ibn-e-Sina Hospital, Faculty of Medicine, Mashhad University of Medical Sciences, Mashhad, Iran

3. Student Research Committee, Mashhad University of Medical Sciences, Mashhad, Iran

4. Golestan Research Center of Psychiatry (GRCP), Golestan University of Medical Sciences, Gorgan, Iran

5. Pharmaceutical Instiute of Technology, Pharmaceutical Research Center, Mashhad University of Medical Sciences, Mashhad, Iran

6. Department of Clinical Pharmacy, School of Pharmacy, Mashhad University of Medical Sciences, Mashhad, Iran metabolic syndrome (1). Hypertension and hypercholesterolemia $(2,3)$, type 2 diabetes, insulin resistance, hyperhomocysteinemia, aging, and obesity (2, 4-6) are among the risk factors for atherosclerosis in which an increase in ADMA has been reported.

In combination with Framingham risk score, the traditional risk factors can be appropriate predictors of cardio-

$\uparrow$ What is “already known” in this topic:

Treatment with atypical antipsychotics can increase traditional risk factors of coronary artery disease, and in many studies clozapine and olanzapine were at the top of the list of the atypical antipsychotics.

\section{$\rightarrow$ What this article adds:}

In clozapine group, levels of non-traditional factors (hsCRP, Homocysteine) and ADMA were significantly higher which indicates these patients are at higher risk of cardiovascular disease. 
vascular events in a group of people, but sometimes they fail to identify people at high risk for cardiovascular events in the near future (7). In other words, some people experience a cardiovascular event, while they are not categorized in high-risk subjects based on Framingham risk score. Therefore, to identify people who are more prone to cardiovascular events, finding serologic biomarkers is essential (8).

In many studies, it was found that higher levels of ADMA is related to higher risks for acute coronary events (1, 8-10).

Metabolic syndrome is a common side effect of atypical antipsychotics and the prevalence of atypical antipsychotic induced metabolic syndrome varies.

Treatment with atypical antipsychotics can increase traditional risk factors of coronary artery disease, and in many studies, clozapine and olanzapine were at the top of the list of the atypical antipsychotics (11-14).

Studies also indicated elevated serum levels of homocysteine and increased metabolic syndrome in patients who receive antipsychotics $(7,15,16)$.

So far, investigations on the prevalence of metabolic syndrome were based on traditional cardiovascular risk factors and few studies have been performed to compare the prevalence of cardiovascular risk factors among atypical antipsychotics based on nontraditional risk factors (17, 18) and there is no study on other cardiovascular biomarkers, like ADMA, in patients receiving different antipsychotics. Also, most of the previous studies consider only one factor (ADMA) in schizophrenic patients $(10,18$, 19 ) or only a group of schizophrenic patients (20), and no study examined a group that discontinued their medication (21). Therefore, in this study, we compares serum levels of ADMA between patients who take two different types of atypical antipsychotics and also a healthy control group. On the other hand, since schizophrenia increases ADMA itself, we consider a group of schizophrenic patient without receiving any drugs.

\section{Methods}

\section{Patients}

In this clinical study, 57 patients with schizophrenia with a history of multiple episodes and 20 healthy voluntaries that fulfilled inclusion and exclusion criteria were entered into the study and were divided into four groups as below:

1. 18 schizophrenic patients received risperidone alone for at least five months.

2. 20 schizophrenic patients received clozapine alone for at least five months.

3. 19 schizophrenic patients that did not receive any antipsychotic for at least five months.

4. 20 healthy voluntaries without any traditional or nontraditional cardiovascular risk factors.

Inclusion criteria consisted of schizophrenic patients receiving the atypical antipsychotic for at least 5 months and not receiving other antipsychotics or drugs with effect on ADMA, in combination with studied antipsychotics. In another study (19) this period of time was considered three months, and we increased into five months. All of their patients experienced more than one relapse (19).

Exclusion criteria consisted of any disease during the study that could distort the results, lack of patient's cooperation to continue studying, unavailability of patients after the inclusion, any hepatic or renal disorders, metabolic syndrome, cancer, infection, chronic inflammatory disease, and any other drug with an effect on ADMA.

\section{Blood sampling and ADMA assay}

After inclusion, the patients' demographic characteristics and history of the disease were recorded in a questionnaire, and $10 \mathrm{~mL}$ blood was collected from them. 5 $\mathrm{mL}$ was used for traditional factors of metabolic syndrome, and the remaining $5 \mathrm{~mL}$ was centrifuged at 1500 $\mathrm{rpm}$, and the serum was removed and stored at $-70{ }^{\circ} \mathrm{C}$. The serum ADMA concentration was measured through ELISA, and CRP and homocysteine levels were measured.

Traditional factors include fasting blood sugar (FBS), HDL cholesterol (mmol/L), low-density lipoprotein (LDL) cholesterol $(\mathrm{mmol} / \mathrm{L})$, triglycerides $(\mathrm{TG})(\mathrm{mmol} / \mathrm{L})$, total cholesterol $(\mathrm{mmol} / \mathrm{L})$, diastolic blood pressure (DBP) (mmHg), systolic blood pressure (SBP) (mmHg), waistline and body mass index. Nontraditional factors include hs-CRP and homocysteine.

\section{Ethical considerations}

The study approved by the Ethics Committee of Mashhad University of Medical Science. Informed consent was obtained from the parents of all patients. In all steps of research, medical confidentiality and privacy were respected.

\section{Statistical analysis}

Data were analyzed using SPSS21. Normality of the quantitative variables was determined using KolmogorovSmirnov test. For comparison of ADMA levels among the four groups, one-way ANOVA and Tukey Post-Hoc tests were carried out. $\mathrm{A} \leq 0.05$ was considered as statistical significance.

\section{Results}

\section{Population study}

In this clinical study, 18 patients were included in the risperidone group, 20 patients in the clozapine group, 19 patients in the schizophrenia group with no medication in the past five months, and 20 patients in the control group (healthy subjects). The age range of samples was 23 to 78 years. $70 \%$ were men, and $30 \%$ were women. The results showed that the mean $\pm \mathrm{SD}$ age was $46.08 \pm 12.54$ years. Descriptive statistics of the traditional, ADMA and nontraditional risk factors in four groups are showed in Table 1.

Comparison of traditional risk factors between groups

According to Table 1 traditional factors including FBS, LDL, DBP, SBP, and TG did not have significant differences between four groups. For other traditional factors containing BMI, Cholesterol, HDL and Waistline Tukey Post Hoc test was done in Table 2. 
Table 1. Comparison of data of traditional and non-traditional factors and ADMA among four groups

\begin{tabular}{|c|c|c|c|c|c|}
\hline Variable & $\begin{array}{l}\text { Risperidone } \\
\text { group }\end{array}$ & $\begin{array}{l}\text { Clozapine } \\
\text { group }\end{array}$ & $\begin{array}{c}\text { Schizophrenic participants } \\
\text { without any drug }\end{array}$ & $\begin{array}{l}\text { Healthy } \\
\text { group }\end{array}$ & $\mathrm{p}^{*} 1$ \\
\hline Fasting blood sugar & $103.06 \pm 10.17$ & $121.40 \pm 40.72$ & $95.11 \pm 12.35$ & $101.65 \pm 15.77$ & 0.06 \\
\hline HDL cholesterol (mmol/L) & $54.06 \pm 10.82$ & $50.50 \pm 14.53$ & $55.79 \pm 13.16$ & $40.50 \pm 10.46$ & 0.001 \\
\hline LDL cholesterol (mmol/L) & $99.50 \pm 19.28$ & $97.20 \pm 19.61$ & $89.63 \pm 26.99$ & $81.40 \pm 26.00$ & 0.075 \\
\hline Triglycerides $(\mathrm{mmol} / \mathrm{L})$ & $118.67 \pm 57.43$ & $147.20 \pm 115.19$ & $95.11 \pm 51.71$ & $99.25 \pm 25.40$ & 0.094 \\
\hline Total cholesterol $(\mathrm{mmol} / \mathrm{L})$ & $178.72 \pm 30.35$ & $177.85 \pm 31.35$ & $158.53 \pm 45.84$ & $145.55 \pm 28.61$ & 0.009 \\
\hline Diastolic blood pressure (mmHg) & $71.39 \pm 6.37$ & $70.50 \pm 6.86$ & $71.32 \pm 8.30$ & $73.75 \pm 7.41$ & 0.534 \\
\hline Systolic blood pressure (mmHg) & $115.28 \pm 7.37$ & $113.75 \pm 8.09$ & $112.89 \pm 10.31$ & $115.75 \pm 7.30$ & 0.693 \\
\hline Waistline & $90.56 \pm 19.15$ & $95.40 \pm 16.07$ & $71.68 \pm 6.73$ & $88.30 \pm 12.00$ & $<0.001$ \\
\hline Body Mass Index & $26.78 \pm 6.79$ & $25.45 \pm 4.83$ & $20.14 \pm 3.08$ & $24.04 \pm 3.42$ & $<0.001$ \\
\hline Homocysteine & $6.64 \pm 3.07$ & $9.21 \pm 3.60$ & $6.72 \pm 2.60$ & $4.49 \pm 1.20$ & $<0.001$ \\
\hline hs-CRP & $2.16 \pm 0.77$ & $3.07 \pm 1.17$ & $1.09 \pm 0.45$ & $0.83 \pm 0.61$ & $<0.001$ \\
\hline ADMA & $1.77 \pm 0.54$ & $2.56 \pm 1.01$ & $1.22 \pm 0.35$ & $1.13 \pm 0.44$ & $<0.001$ \\
\hline
\end{tabular}

Data are presented as mean $\pm \mathrm{SD}$

*One-way ANOVA

The results presented in Table 2 indicate that, BMI has a significant difference in schizophrenic patients without any drug compared with risperidone and clozapine group. According to Table 1, BMI is lower in schizophrenic participants without any drug group.

HDL has a significant difference in the healthy control group compared with risperidone group and schizophrenic participants without any drug group. According to Table $1, \mathrm{HDL}$ is lower in the healthy group.

Cholesterol has a significant difference in the healthy group compared with risperidone and clozapine group. According to Table 1, Cholesterol is considerably lower in the healthy group.

Waistline is significantly lower in schizophrenic participants without any drug compared with other three groups.

The results presented in Table 3 showed that: Homocysteine is significantly different in the clozapine group compared with the other three groups, and according to Table 1 , it is considerably higher in the clozapine group.
hs-CRP has a significant difference in the clozapine group compared with the other three groups, and according to Table 1 , it is considerably higher in the clozapine group. Also, hs-CRP is significantly different in risperidone group in comparison with the other three groups, and according to Table 1, it is noticeably higher than schizophrenic participants without any drug group and the healthy group. Also, ADMA is significantly different in the clozapine group compared with the other three groups, and according to Table 1, it is considerably higher in the clozapine group. Moreover, ADMA and non-traditional variables have significant differences between the four groups (Table 1). Serum concentrations of ADMA were compared in groups. In the clozapine group compared to three other groups all three factors (ADMA, hs-CRP, Homocysteine) were significantly higher $(p \leq 0.05)$. Also, hs$\mathrm{CRP}$ in the group receiving risperidone compared to the control group and group without treatment was significantly higher $(\mathrm{p} \leq 0.05)$ (Table 3$)$.

Table 2. Comparison of traditional factors among four groups

\begin{tabular}{|c|c|c|c|c|c|}
\hline Group & & BMI & Cholesterol & $\mathrm{HDL}$ & Waistline \\
\hline \multirow[t]{3}{*}{ Risperidone group } & Nodrug & $<0.001 *(2.45 \text { to } 10.84)^{* *}$ & $0.44(-13.08$ to 53.47$)$ & $0.99(-13.11$ to 9.65$)$ & $0.001(6.46$ to 31.28$)$ \\
\hline & Healthy & $0.35(-1.39$ to 6.88$)$ & $0.04(0.31$ to 66.04$)$ & $0.01(2.32$ to 24.80$)$ & $0.98(-10.0$ to 14.51$)$ \\
\hline & Cloz & $0.89(-2.80$ to 5.47$)$ & $1.00(-31.99$ to 33.74$)$ & $0.90(-7.68$ to 14.80$)$ & $0.80(-17.10$ to 7.41$)$ \\
\hline \multirow[t]{3}{*}{ Clozapine group } & Nodrug & $0.004(1.22$ to 9.32$)$ & $0.46(-13.08$ to 51.73$)$ & $0.67(-16.37$ to 5.79$)$ & $<0.001$ (11.63 to 36.80$)$ \\
\hline & Ris & $0.89(-5.47$ to 2.80$)$ & $0.99(-33.74$ to 31.99$)$ & $0.90(-14.80$ to 7.68$)$ & $0.80(-7.41$ to 17.10$)$ \\
\hline & Healthy & $0.86(-2.62$ to 5.44$)$ & $0.04(0.31$ to 64.29$)$ & $0.09(-0.94$ to 20.94$)$ & $0.46(-4.83$ to 19.03$)$ \\
\hline \multirow{6}{*}{$\begin{array}{l}\text { Schizophrenic } \\
\text { participants without } \\
\text { any drug } \\
\text { Healthy group }\end{array}$} & Healthy & $0.06(-7.98$ to 0.18$)$ & $0.79(-19.43$ to 45.38$)$ & $0.002(4.21$ to 26.37$)$ & $0.002(-28.70$ to 4.53$)$ \\
\hline & Ris & $<0.001(-10.84$ to -2.45$)$ & $0.44(-53.47$ to 13.08$)$ & $0.99(-9.65$ to 13.11$)$ & $0.001(-31.26$ to -6.46$)$ \\
\hline & Cloz & $0.004(-9.39$ to -1.22$)$ & $0.46(-51.73$ to 13.08$)$ & $0.67(-5.79$ to 16.37$)$ & $<0.001(-35.80$ to -11.63$)$ \\
\hline & Nodrug & $0.06(-0.18$ to 7.09$)$ & $0.79(-45.38$ to 19.43$)$ & $0.002(-26.37$ to -4.21$)$ & $0.002(4.53$ to 28.70$)$ \\
\hline & Ris & $0.35(-6.88$ to 1.39$)$ & $0.04(-66.04$ to -0.31$)$ & $0.01(-24.80$ to 2.32$)$ & $0.98(-14.51$ to 10.00$)$ \\
\hline & Cloz & $0.86(-5.44$ to 2.62$)$ & $0.04(-64.29$ to -0.31$)$ & $0.09(-20.94$ to 0.94$)$ & $0.46(-19.03$ to 4.83$)$ \\
\hline
\end{tabular}

Table 3. Comparison of ADMA and non-traditional factors among four groups

\begin{tabular}{|c|c|c|c|c|}
\hline Group & & Homocysteine & hs-CRP & ADMA \\
\hline \multirow[t]{3}{*}{ Risperidone group } & Nodrug & $0.99 *(-2.52$ to 2.37$) * *$ & $0.01(0.16$ to 1.96$)$ & $0.16(-0.12$ to 1.22$)$ \\
\hline & Healthy & $0.10(-0.26$ to 4.56$)$ & $0.001(0.44$ to 2.21$)$ & $0.07(0.03$ to 1.30$)$ \\
\hline & $\mathrm{Cloz}$ & $0.03(-4.98$ to -0.14$)$ & $0.04(-1.80$ to -0.02$)$ & $0.01(-1.46$ to -0.12$)$ \\
\hline \multirow[t]{3}{*}{ Clozapine group } & Nodrug & $0.03(0.10$ to 4.87$)$ & $<0.001$ (1.10 to 2.85$)$ & $<0.001(0.68$ to 2.00$)$ \\
\hline & Ris & $0.03(0.14$ to 4.98$)$ & $0.04(0.02$ to 1.80$)$ & $0.01(0.12$ to 1.46$)$ \\
\hline & Healthy & $<0.001(2.36$ to 7.06$)$ & $<0.001(1.37$ to 3.10$)$ & $<0.001$ (0.77 to 2.08$)$ \\
\hline Schizophrenic participants & Healthy & $0.07(-0.15$ to 4.61$)$ & $0.92(-0.61$ to 1.13$)$ & $0.99(-0.57$ to 0.74$)$ \\
\hline \multirow{2}{*}{ without any drug } & Ris & $1.00(-2.37$ to 2.52$)$ & $0.01(-1.96$ to -0.16$)$ & $0.16(-1.22$ to 0.12$)$ \\
\hline & Cloz & $0.03(-4.87$ to -0.10$)$ & $<0.001(-2.85$ to -1.10$)$ & $<0.001(-2.00$ to 0.68$)$ \\
\hline \multirow[t]{3}{*}{ Healthy group } & Nodrug & $0.07(-4.61$ to 0.15$)$ & $0.92(-1.13$ to 0.61$)$ & $0.99(-0.74$ to 0.57$)$ \\
\hline & Ris & $0.10(-4.56$ to 0.26$)$ & $0.001(-2.21$ to -0.44$)$ & $0.07(-1.30$ to 0.03$)$ \\
\hline & Cloz & $<0.001(-7.06$ to -2.36$)$ & $<0.001(-3.10$ to -1.37$)$ & $<0.001(-2.08$ to -0.77$)$ \\
\hline
\end{tabular}

${ }^{*} \mathrm{p},{ }^{* * 95 \% \mathrm{CI}}$ 


\section{Discussion}

This study is the first study comparing ADMA, traditional and non-traditional factors between patients who take two types of atypical antipsychotics in comparison with a control group. The results showed that the serum level of ADMA was significantly higher in schizophrenic patients who take clozapine than all of the other groups and there was no significant difference between schizophrenic patients without treatment and the healthy group. Also, there was no significant difference between the risperidone group and the other groups (except for the clozapine group) in ADMA level.

Nitric oxide is a free radical with some effects: vasodilation, antithrombosis, and inhibition of clot formation; and ADMA is a competitive inhibitor of nitric oxide synthetase and a vascular injury biomarker. So, we can say that clozapine injures the vascular endothelium more than other antipsychotics and according to previous studies, clozapine causes atherosclerosis more than other antipsychotics (20). Thus clozapine causes vascular injury and so can increase ADMA that is similar to our results.

In this study the groups matched in terms of ADMA increasing factors as much as possible. On the other hand, because schizophrenia increases ADMA itself, we consider a group with schizophrenia and without any drug (group 3). So if we see different ADMA levels in groups, it is of high probability because of drugs.

In a study on schizophrenic patients, ADMA was clearly higher in the group with the history of several episodes than the first episode (19). So, we selected patients for all of the groups from the patients with several episodes.

In another study, ADMA level in patients with the first episode was significantly increased compared to the control group. ADMA was measured again after two months of treatment with antipsychotics which showed a significant decrease compared to the initial level. There was no relationship between the level of ADMA and the severity of disease $(22,23)$. So we consider a no-drug schizophrenic group, but in our study, there was no significant difference between no-drug patients and healthy group.

There was also a statistically significant difference between the control group compared to clozapine receiving schizophrenia patients and the no-drug group in terms of ADMA, and this is consistent with the study of Das (24), Celik (19), and Zincir (22). However, the study of Jorgensen et al. (14) showed no difference between various groups.

The results of this study showed that patients with schizophrenia treated with clozapine had a higher ADMA than the control group. Furthermore, ADMA level was reduced in schizophrenic patients group that did not consume any drug during the last 5 months. This is also similar to the study of Celik et al. $(9,19)$.

In another study, short-term treatment initially creates a protection against endothelial damage which may be due to reduced agitation and activity of the autonomic nervous system, but no significant association was found between the disease severities (9). This study showed that drug consumption is a factor which increases possible risk factors in heart disease (14).
In our study, homocysteine in schizophrenic patients who take clozapine was significantly higher than other groups, and there was no significant difference between schizophrenic patients without treatment and healthy group. Also, there was no significant difference between the risperidone group and other groups (except clozapine group) in homocysteine level.

These results are not consistent with those of Adam Wysokin'ski study (20), but was similar to the study of Shusuke Numata et al. (25). It seems that homocysteine reducing strategies are effective in patients with schizophrenia particularly those receiving clozapine, and this reduction has been proven through consumption of folate, B12, and pyridoxine. Reduction of this factor is effective in reducing the risk of cardiovascular diseases in patients with schizophrenia $(18,25,26)$.

Some studies have indicated higher levels of homocysteine and risk of metabolic syndrome in patients receiving antipsychotics, that is similar to our study $(7,15,16)$.

In our study, hs-CRP in patients who take clozapine was significantly higher than other groups. hs-CRP in patients who take risperidone was significantly higher than the healthy group and schizophrenic without treatment group. There was no significant difference between healthy group and patients without treatment group in hs-CRP level.

A study was carried out in 2010 on the effects of haloperidol, risperidone, and olanzapine antipsychotics on CRP. Three months after receiving the drug, CRP level in patients receiving haloperidol was $92.7 \%$ higher than risperidone receiving patients (17). However, in our study among the investigated atypical antipsychotics, clozapine was associated with a significantly higher CRP than risperidone.

In our study, there were some statistically significant differences between schizophrenia patients and the control group in terms of traditional factors of FBS, BMI, cholesterol, LDL, and waistline that is similar to the study of Błażej Misiak (27). In addition, the drug-receiving groups had higher total cholesterol levels than the other two groups, and this was also consistent with the study of Błażej Misiak (27).

Generally, in our study, there was a little significant difference in traditional factors among our groups. It is probably because of the exclusion criteria as we excluded patients with metabolic syndrome.

\section{Conclusion}

The traditional factors including High-density lipoprotein, total cholesterol, waistline, and Body mass index, and non-traditional factors including Homocysteine, hs-CRP and ADMA were higher in patients with schizophrenia compared to healthy group ( $\mathrm{p}$-value $\leq 0.05$ ). Moreover, in the clozapine group, all mentioned non-traditional factors and ADMA were significantly higher than other groups $(\mathrm{p} \leq 0.05)$.

\section{Limitation}

We couldn't match the mean ages of the groups. Due to the fact that the patients had chronic schizophrenia for a long time, we couldn't match the groups in terms of dos- 
age and duration of treatment for each drug.

\section{Acknowledgments}

The authors would like to thank Pharmacologic Sciences Research Center of Ferdowsi University and Psychiatry and Behavioural Sciences Research Center of Ibn-e-Sina Hospital. Voluntary participation and cooperation of the medical students in the study is highly appreciated.

\section{Funding}

This work was extracted from residency thesis in the Mashhad University of medical science and without any organizational financial support.

\section{Conflict of Interests}

The authors declare that they have no competing interests.

\section{References}

1. Maeda T, Yoshimura T, Okamura H. Asymmetric dimethylarginine, an endogenous inhibitor of nitric oxide synthase, in maternal and fetal circulation. J Soc Gynecol Investig. 2003;10(1):2-4.

2. Panza JA, Quyyumi AA, Brush Jr JE, Epstein SE. Abnormal endothelium-dependent vascular relaxation in patients with essential hypertension. N Engl J Med. 1990;323(1):22-7.

3. Celermajer D, Sorensen K, Georgakopoulos D, Bull C, Thomas O, Robinson J, et al. Cigarette smoking is associated with dose-related and potentially reversible impairment of endothelium-dependent dilation in healthy young adults. Circulation. 1993;88(5):2149-55.

4. Mäkimattila S, Liu ML, Vakkilainen J, Schlenzka A, Lahdenperä S, Syvänne M, et al. Impaired endothelium-dependent vasodilation in type 2 diabetes. Relation to LDL size, oxidized LDL, and antioxidants. Diabetes Care. 1999;22(6):973-81.

5. Tawakol A, Omland T, Gerhard M, Wu JT, Creager MA. Hyperhomocyst (e) inemia is associated with impaired endotheliumdependent vasodilation in humans. Circulation. 1997;95(5):1119-21.

6. Hingorani AD, Cross J, Kharbanda RK, Mullen MJ, Bhagat K, Taylor $\mathrm{M}$, et al. Acute systemic inflammation impairs endotheliumdependent dilatation in humans. Circulation. 2000;102(9):994-9.

7. Vuksan-Ćusa B, Jakovljević M, Šagud M, Peleš AM, Marčinko D, Topić R, et al. Metabolic syndrome and serum homocysteine in patients with bipolar disorder and schizophrenia treated with second generation antipsychotics. Psychiatry Res. 2011;189(1):21-5.

8. Böger RH. Asymmetric dimethylarginine, an endogenous inhibitor of nitric oxide synthase, explains the "L-arginine paradox" and acts as a novel cardiovascular risk factor. J Nutr. 2004;134(10):2842S-7S.

9. Nonaka-Hashida S, Sekine M, Ozeki Y, Fujii K, Akiyama K, Shimoda K, et al. Plasma concentrations of three methylated arginines, endogenous nitric oxide synthase inhibitors, in schizophrenic patients undergoing antipsychotic drug treatment. Psychiatry Res. 2016;238:203-10.

10. Jorgensen A, Knorr U, Soendergaard MG, Lykkesfeldt J, FinkJensen A, Poulsen HE, et al. Asymmetric dimethylarginine in somatically healthy schizophrenia patients treated with atypical antipsychotics: a case-control study. BMC Psychiatry. 2015;15(1):67.

11. Said MA, Sulaiman AH, Habil MH, Das S, Bakar A, Yusoff RM, et al. Metabolic syndrome and cardiovascular risk among patients with schizophrenia receiving antipsychotics in Malaysia. Singapore Med J. 2012;53(12):801.

12. Newcomer JW. Second-generation (atypical) antipsychotics and metabolic effects. CNS Drugs. 2005;19(1):1-93.

13. Kritharides L, Rye KA, Lambert TJ, Jessup W. Lipidology, cardiovascular risk, and schizophrenia. Curr Opin Lipidol. 2016;27(3):305-7.

14. Jorgensen A, Knorr U, Soendergaard MG, Lykkesfeldt J, FinkJensen A, Poulsen HE, et al. Asymmetric dimethylarginine in somatically healthy schizophrenia patients treated with atypical antipsychotics: a case-control study. BMC Psychiatry. 2015;15(1):1.

15. Malhotra N, Kulhara P, Chakrabarti S, Grover S. Lifestyle related factors \& impact of metabolic syndrome on quality of life, level of functioning \& self-esteem in patients with bipolar disorder \& schizophrenia. Indian J Med Res. 2016;143(4):434.

16. Malan-Müller S, Kilian S, van den Heuvel LL, Bardien S, Asmal L, Warnich L, et al. A systematic review of genetic variants associated with metabolic syndrome in patients with schizophrenia. Schizophr Res. 2016;170(1):1-17.

17. Diaz FJ, Pérez-Iglesias R, Mata I, Martínez-Garcia O, VázquezBarquero JL, de Leon J, et al. Possible effects of some antipsychotic drugs on C-reactive protein in a drug-naïve psychotic sample. Schizophr Res. 2010;121(1):207-12.

18. Levine J, Stahl Z, Sela B-A, Ruderman V, Shumaico O, Babushkin I, et al. Homocysteine-reducing strategies improve symptoms in chronic schizophrenic patients with hyperhomocysteinemia. Biol Psychiatry. 2006;60(3):265-9.

19. Celik C, Cayci T, Ozdemir B, Akgul EO, Zincir S, Balikci A, et al. Plasma asymmetric dimethylarginine (ADMA) concentrations in patients with first and multiple episode schizophrenia. Psychiatry res. 2011;190(2):177-80.

20. Wysokiński A, Kłoszewska I. Homocysteine levels in patients with schizophrenia on clozapine monotherapy. Neurochem Res. 2013;38(10):2056-62.

21. Dashti S, Aboutaleb N, Shahbazi A. The effect of leptin on prepulse inhibition in a developmental model of schizophrenia. Neurosci Lett. 2013;555:57-61.

22. Zincir S. Asymmetric dimethylarginine (ADMA) and treatment response relationship in male patients with first-episode schizophrenia: Author' s reply. Psychiatry Res. 2015;2(227):371.

23. Zincir S, Zincir SB, Doruk A, Erdem M, Celik C, Ak M, et al. Asymmetric dimethylarginine (ADMA) and treatment response relationship in male patients with first-episode schizophrenia: A controlled study. Psychiatry Res. 2014;220(1):76-80.

24. Das I, Khan N, Puri B, Hirsch S. Elevated endogenous nitric oxide synthase inhibitor in schizophrenic plasma may reflect abnormalities in brain nitric oxide production. Neurosci Lett. 1996;215(3):209-11.

25. Numata S, Kinoshita M, Tajima A, Nishi A, Imoto I, Ohmori T. Evaluation of an association between plasma total homocysteine and schizophrenia by a Mendelian randomization analysis. BMC Med Genet. 2015;16(1):54.

26. Roffman JL, Lamberti JS, Achtyes E, Macklin EA, Galendez GC, Raeke LH, et al. Randomized multicenter investigation of folate plus vitamin B12 supplementation in schizophrenia. JAMA Psychiatry. 2013;70(5):481-9.

27. Misiak B, Frydecka D, Łaczmański Ł, Ślęzak R, Kiejna A. Effects of second-generation antipsychotics on selected markers of onecarbon metabolism and metabolic syndrome components in firstepisode schizophrenia patients. Eur J Clin Pharmacol. 2014;70(12):1433-41. 